\title{
Fault Tolerant Modular Inductive Power Transfer System Design Using Resonator Coil
}

\author{
Hakan Polat, Enes Ayaz, Ogün Altun, Ozan Keysan
}

\begin{abstract}
In this paper, a design for an inductive power transfer system with a series-series topology with an intermediate resonator is discussed. The proposed method is an improved method that is derived from a conventional IPT system design. While it is applicable for a wide range of different operations, a contactless slip ring design is the main system under investigation. The idea behind adding a resonator coil is to achieve fault tolerance where the system can operate under various open-circuited fault conditions. The proposed system is a two transmitter and four receiver with an intermediate resonator system where the $R x$ modules are connected parallel to a common DC-bus. The system is found to be fault-tolerant to $\mathrm{Rx}$ side open circuit faults. For normal operation at rated power, $90.6 \%$ efficiency was achieved.
\end{abstract}

Index Terms-Wireless power transfer, inductive power transfer, modular design, three-coil design, common DC bus, fault tolerance, reliability

\section{INTRODUCTION}

Inductive power transfer (IPT) systems are becoming more popular in recent years. Although a typical IPT system has a transmitter coil and a receiver coil, the multi-coil system has gained popularity thanks to its advantages such as increased transmission distance, misalignment tolerance, and fault tolerance [1], [2]. A multi-transmitter system provides flexible mobility to a receiver, and it is suitable for portable chargers. Also, the multi-transmitter system is used to avoid misalignment that decreases the efficiency and power transfer ratings [3]. A multi-receiver system contributes to EV chargers to flexible charging at parking. Besides, selective load design can be implemented by using the multi-receiver system with a different resonant frequency [4]-[6]. A resonator coil, called third-coil, is used to obtain an IPT system, tolerant to misalignment.

The aim of this paper is to design a contactless rotational IPT system that can replace conventional slip-rings and brushless field exciters of large synchronous machines. Slip-rings are prone to wear due to constant friction. Therefore, regular maintenance is necessary, which increases the operation cost. Brushless exciters are often permanent

(C)2021 IEEE. Personal use of this material is permitted. Permission fromIEEE must be obtained for all other uses, in any current or future media,including reprinting/republishing this material for advertising or promotionalpurposes, creating new collective works, for resale or redistribution to serversor lists, or reuse of any copyrighted component of this work in other works. Under Review.

H. Polat, Enes Ayaz, Ogün Altun and Ozan Keysan are with Middle East Technical University, Department of Electrical and Electronics Engineering, Ankara, Turkey. Email: keysan@metu.edu.tr

Corresponding Author: Ozan Keysan, keysan@metu.edu.tr magnet synchronous machines (PMSM) where the induced voltage on the rotor winding is used to control the field current. However, at low rotating speeds, control of the field current is problematic due to reduced induced voltage. The proposed system aims to eliminate wear using IPT systems and achieve a speed-independent operation while satisfying high fault tolerance and reliability requirements.

In conventional series-series IPT systems, light or opencircuited load conditions are problematic due to dangerously high transmitter currents. A possible solution to improve tolerance against these faults is to employ modularity where continuous operation is achieved by increasing the number of $\mathrm{Rx}$ modules coupled to a single $\mathrm{Tx}$ coil. During a single $\mathrm{Rx}$ module open circuit fault, there is still enough magnetic coupling between the $\mathrm{Tx}$ coil and the remaining $\mathrm{Rx}$ modules. In the literature, series-series/parallel common DC bus connected systems are investigated in terms of current balancing [7]. In [8], a $2 \mathrm{Tx}-4 \mathrm{Rx}$ series-series compensated parallel-connected common DC-bus system was presented where the current unbalance was significantly reduced using additional cross-coupling between the Rx modules. Another proposed method was the intentional detuning of $\mathrm{Rx}$ side resonant frequency, which further enhances current balancing [8]. However, it is not applicable to series-parallel topology as stated in [7]. A major problem of existing modular systems is the loss of current balancing under faulty conditions due to differences in their magnetic couplings.

This study proposes the addition of middle stage resonator to $2 \mathrm{Tx}-4 \mathrm{Rx}$ where equal $\mathrm{Rx}$ module power sharing is achieved under various open-circuit fault conditions. Moreover, in this paper, a conventional bifurcation free series-series IPT system design given in [9] is extended to be applicable to systems with middle stage resonators. The analytical approach is compared with the simulation results, and a $1 \mathrm{~kW}$ experimental prototype is built. The experiments are performed under different fault conditions, and the fault tolerance of the proposed system is investigated.

The proposed $2 \mathrm{Tx}-4 \mathrm{Rx}$ system with middle stage resonator is presented in Fig. 1. The power is transfered from a stationary $\mathrm{Tx}$ side to a rotating resonator and rotating $\mathrm{Rx}$ modules. The advantages of adding a middle stage resonator coil may be listed as follows:

- Regardless of the current phase and magnitude of the Tx coils, a single sinusoidal current passes through the 


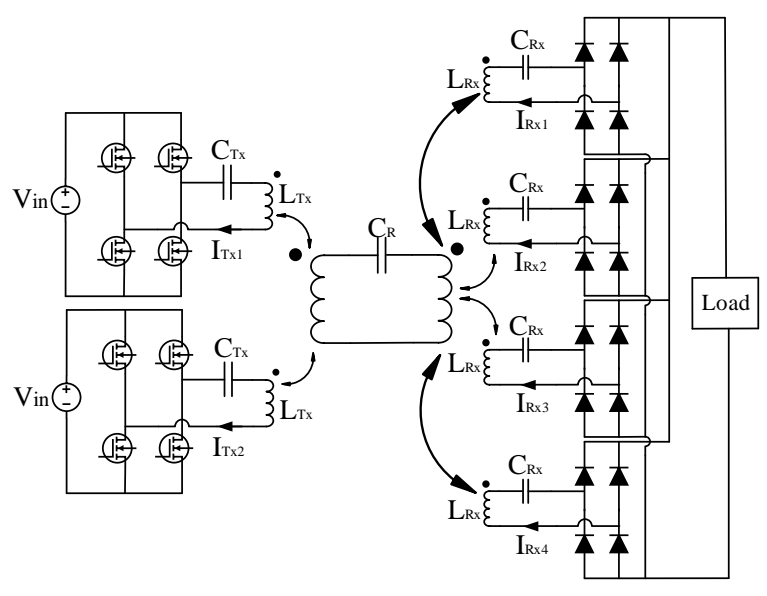

Fig. 1. The proposed $2 \mathrm{Tx}-4 \mathrm{Rx}$ system with resonator circuit.

resonator coil which allows flexible control over the power transfer.

- The sinusoidal current of the resonator creates a constant magnetic flux density, which results in equal coupling between each Rx module and the resonator.

- In case of an open circuit fault in the Rx side, the air gap flux distribution is not disturbed, and hence the coupling between all Rx modules and the resonator coil stays constant. Therefore, normal operation can still be achieved naturally without any complex control techniques. A similar statement can also be made for open circuited $\mathrm{Tx}$ fault where power can still be delivered through a single Tx module.

\section{Design Methodology}

The proposed system structure is presented in Fig. 1. It consists of $2 \mathrm{Tx}$ and $4 \mathrm{Rx}$ modules. In the proposed system, the Tx and Rx side are decoupled. The power is transferred with a resonator coil placed between the Tx and Rx coils. The

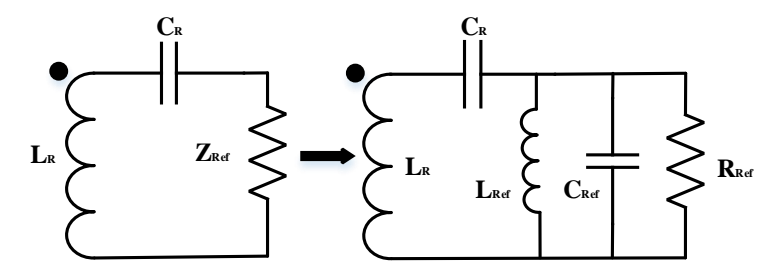

Fig. 2. Reflected impedance from $\mathrm{Rx}$ side to resonator side.

reflected impedance model from $\mathrm{Rx}$ side to resonator side is presented in Fig. 2. The reflected impedance $Z_{\text {ref }}$ is given in (1).

$$
Z_{r e f}=\frac{\omega^{2} M_{R, R x}^{2}}{j \omega L_{R x}+\frac{1}{j \omega C_{R x}}+R_{L}}
$$

The reflected parallel resonance circuit impedance can be written as in (2).

$$
Z_{\text {ref }}=\frac{1}{\frac{1}{j \omega L_{\text {ref }}}+j \omega C_{\text {ref }}+\frac{1}{R_{\text {ref }}}}
$$

By using (1) and (2) $L_{r e f}, C_{r e f}$ and $R_{r e f}$ can be found as in (3) where $\Phi=\omega^{2} M_{R, R x}^{2}$.

$$
L_{r e f}=\Phi C_{R x}, C_{r e f}=\frac{L_{R x}}{\Phi}, R_{r e f}=\frac{\Phi}{R_{L}}
$$

The parallel resonant impedance $L_{\text {ref }} \| C_{\text {ref }}$ act as an open circuit at the vicinity of the resonant frequency. However, it is beneficial to have a low quality factor at the parallel resonant circuit since an open-circuited $L_{\text {ref }} \| C_{\text {ref }}$ results in a single resistive component, and the circuit can be reduced to a conventional SS topology. To achieve a low quality factor at the parallel reflected resonant circuit, $L_{\text {ref }}$ should be high, which means that $C_{R x}$ should also be selected high. The quality factor of the $\mathrm{Rx}$ side $\left(Q_{R x}\right)$ is given in (4). The internal resistance of the Rx side IPT coil is neglected since it is often negligibly smaller than the load resistance.

$$
Q_{R x}=\frac{\omega L_{R x}}{R_{L}}=\frac{1}{\omega C_{R x} R_{L}}
$$

From (4), it can be derived that the $Q_{R x}$ should be selected low, which means a lower inductance value should be selected for the Rx side. However, a very low $Q_{R x}$ is not desired since it increases the required inductance of the resonator and its size. A low quality factor can be compensated by increasing the coupling between the Rx and resonator coils. However, for some applications increasing the coupling may not always be feasible.

The analysis so far shows that under low $Q_{R x}$ condition, conventional series-series design methods can be used. Aditya et al. proposed a method where the bifurcation phenomenon is eliminated during the design procedure [9]. The proposed method follows a similar approach. Using the first harmonic approximation (FHA) and under the assumption of $100 \%$ efficiency, initial system parameters can be defined as in Table I.

TABLE I

SYSTEM PARAMETERS

\begin{tabular}{ll} 
Parameters & Value \\
\hline Power Rating & $500 \mathrm{~W}$ \\
Input Voltage $_{\text {in }}$ & $100 \mathrm{~V}_{D C}$ \\
Output VoltageV $_{\text {out }}$ & $100 \mathrm{~V}_{D C}$ \\
Resonant Frequency & $150 \mathrm{kHz}$ \\
${\text { Load Resistance } \mathrm{R}_{\mathrm{L}}}$ & $20 \Omega$ \\
Input Voltage $_{\text {in,rms }}$ & $90 V_{r m s}$ \\
Output Voltage $\mathrm{V}_{\text {out }}$ & $100 V_{r m s}$ \\
Input Current $\mathrm{I}_{\mathrm{Tx}}$ & $5.55 A_{r m s}$ \\
Output Current $\mathrm{I}_{\mathrm{Rx}}$ & $5 A_{r m s}$ \\
\hline
\end{tabular}

An initial selection should be made for the percentage $(\Psi)$ of the resonator current to the Tx current as in (5).

$$
\Psi=\frac{I_{R}}{I_{T x}}=1
$$

From (5) $I_{R}$ can be found as $5.55 A_{r m s}$. At the resonant frequency, the induced voltage on the Tx coil is equal to the 
input voltage. Hence the mutual inductance between Tx and resonator coils can be calculated as in (6).

$$
M_{T x, R}=\frac{V_{i n}}{\omega_{0} I_{R}}=15.62 \mu H
$$

A similar approach can be made for the mutual inductance between resonator and $\mathrm{Rx}$ side coil as in (7).

$$
M_{R x, R}=\frac{V_{\text {out }}}{\omega_{0} I_{R}}=17.36 \mu H
$$

At this stage, two parameters should be selected, which are the coupling factors between Tx-resonator $\left(k_{T x, R}\right)$ and resonator$\mathrm{Rx}$ side $\left(k_{R x, R}\right)$. A selection of relatively low quality $\mathrm{Rx}$ side is necessary, as discussed before. Since our load resistance is constant during operation, low $\mathrm{Rx}$ side inductance should be selected. A selection of $Q_{R x}=1.5$ and $k_{R x, R}=0.4$ is found to be suitable for our design. High coupling between resonator and $\mathrm{Rx}$ side means that the airgap is small between these coils. Hence, the Rx side inductance can be calculated as in (8) where $R_{e q u}$ is the resistance seen from the diode rectifier in the $\mathrm{Rx}$ side.

$$
L_{R x}=\frac{Q_{R x} R_{e q u}}{\omega_{0}}=25.47 \mu H
$$

Since the $k_{R x, R}=0.4$ was selected, it is now possible to determine the resonator inductance as in (9).

$$
L_{R}=\frac{M_{R x, R}}{k_{R x, R}^{2} L_{R x}}=73.98 \mu H
$$

At this stage, the equivalent load resistance $\left(R_{\text {equ }}\right)$ can be reflected to the resonator side to find the quality factor of the resonator $\left(Q_{R}\right)$ given in (10).

$$
Q_{R}=\frac{\omega_{0} L_{R}}{R_{\text {ref }}}=\frac{\omega_{0} L_{R}}{\frac{\omega_{0}^{2} M_{R x, R}^{2}}{R_{\text {equ }}}}=4.167
$$

In order to achieve a bifurcation free operation the critical coupling formula presented in [9] is used. The critical coupling $\left(k_{c}\right)$ is found in (11).

$$
k_{c}=\frac{1}{Q_{R}} \sqrt{1-\frac{1}{4 Q_{R}^{2}}}=0.2382
$$

It is important to note that this approximation is only valid when the $Q_{R x}$ is selected low and/or $k_{R x, R}$ is selected to be high. A selection of 0.2 for the coupling between Tx and resonator $\left(k_{T x, R}\right)$ is found to be suitable for bifurcation free operation. Finally, the Tx side coil inductance can be calculated as in (12).

$$
L_{T x}=\frac{M_{T x, R}}{k_{T x, R}^{2} L_{R}}=82.51 \mu H
$$

All three coils are adjusted to have the same resonant frequency and hence the $C_{T x}, C_{R}$ and $C_{R x}$ can be calculated as in (13).

$$
\begin{array}{r}
C=\frac{1}{L \omega_{0}^{2}}, C_{T x}=13.6 n F \\
C_{R}=15.21 n F, C_{R x}=44.2 n F
\end{array}
$$

The presented methodology is appliable for various applications ranging from small power applications to large power

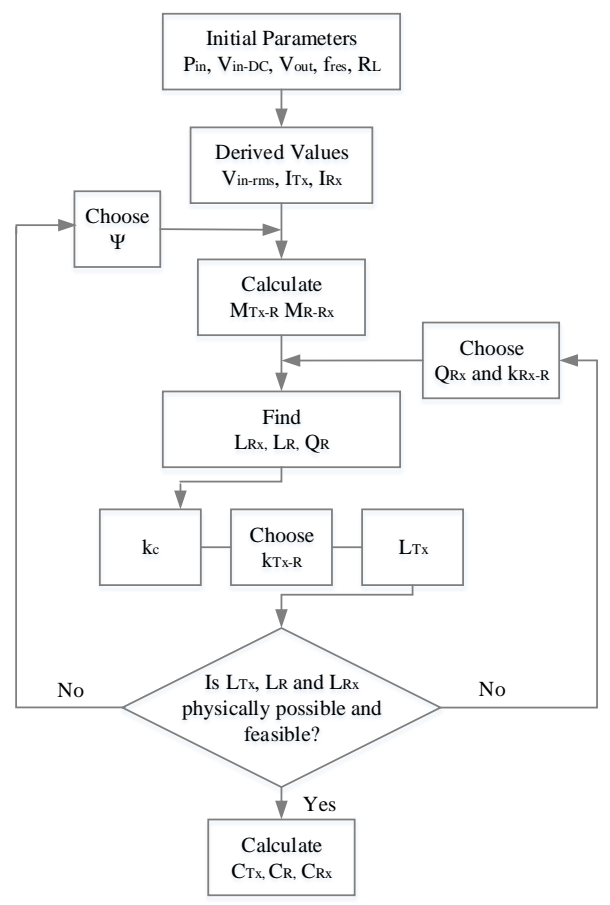

Fig. 3. Flowchart of the proposed design.

applications like electric vehicle IPT chargers by using the generalized design flow-chart is given in Fig. 3. The finalized system parameters for the contactless slip ring design are presented in Table II.

TABLE II

FINAL SYSTEM PARAMETERS

\begin{tabular}{ll} 
Parameters & Contactless Slip Ring \\
\hline Resonant Frequency & $150 \mathrm{kHz}$ \\
$\Psi$ & 1 \\
$\mathrm{~L}_{\mathrm{Tx}}$ & $82.51 \mu \mathrm{H}$ \\
$\mathrm{L}_{\mathrm{R}}$ & $73.98 \mu \mathrm{H}$ \\
$\mathrm{L}_{\mathrm{Rx}}$ & $25.47 \mu H$ \\
$\mathrm{C}_{\mathrm{Tx}}$ & $13.60 \mathrm{nF}$ \\
$\mathrm{C}_{\mathrm{R}}$ & $15.21 \mathrm{nF}$ \\
$\mathrm{C}_{\mathrm{Rx}}$ & $44.20 \mathrm{nF}$ \\
$\mathrm{M}_{\mathrm{Tx}, \mathrm{R}}$ & $15.62 \mu \mathrm{H}$ \\
$\mathrm{M}_{\mathrm{Rx}, \mathrm{R}}$ & $17.36 \mu \mathrm{H}$ \\
$\mathrm{k}_{\mathrm{Tx}}, \mathrm{R}$ & 0.2 \\
$\mathrm{k}_{\mathrm{Rx}, \mathrm{R}}$ & 0.4 \\
\hline
\end{tabular}

The initial assumption of a low $Q_{R x}$ allowed us to use of conventional IPT design as proposed in [9]. However, the feasibility of the assumption should be checked. In Fig. 4 the critical coupling of the analytical design and simulations are given. The design is repeated with a higher $Q_{R x}$, and the analytical $k_{c}$ value are given. The $L_{T x}$ is calculated using the analytical critical coupling value. For a low $Q_{R x}$ assumption, the analytical design gives successful limitations for bifurcation free operation. Whereas simulation limits deviate from analytical design as $Q_{R x}$ increases. The increase of $Q_{R x}$ also increases the sizing of the Rx side IPT coil whereas, the resonator and Tx side coil sizes decreases. 


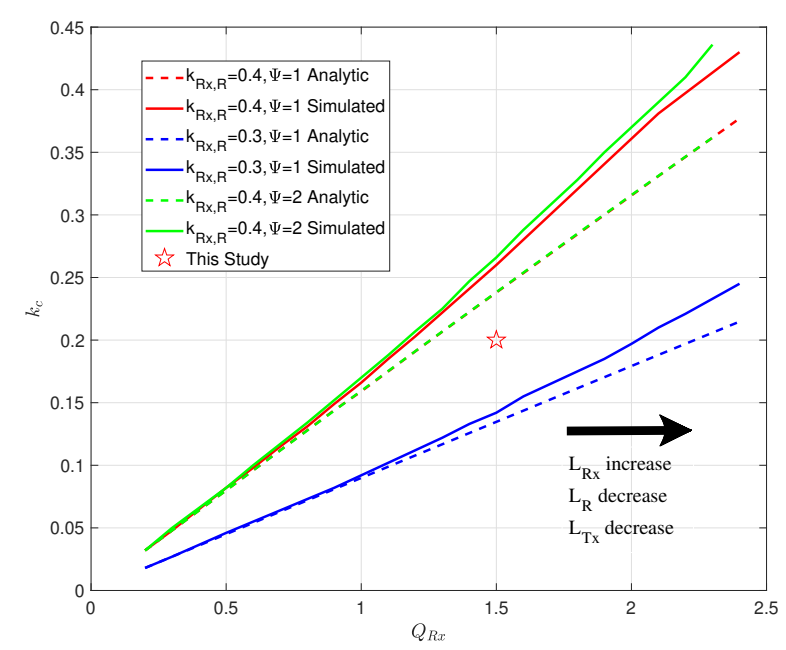

Fig. 4. Comparison of analytical and simulated critical coupling for different design parameters.

\section{EXPERIMENTAL RESULTS}

\section{A. Experimental Setup}

To verify the proposed system, a $1 \mathrm{~kW}$ prototype is built with $2 \mathrm{Tx}-4 \mathrm{Rx}$ module and a single middle stage resonator as shown in Fig. 5. The experimental setup parameters are listed in Table III. Unlike most IPT systems, the air gap of the IPT system is under the designer's control for the contactless slip ring design. However, as stated previously, fault tolerance is the key for reliable operation, which is the main motivation behind adding a resonator coil.

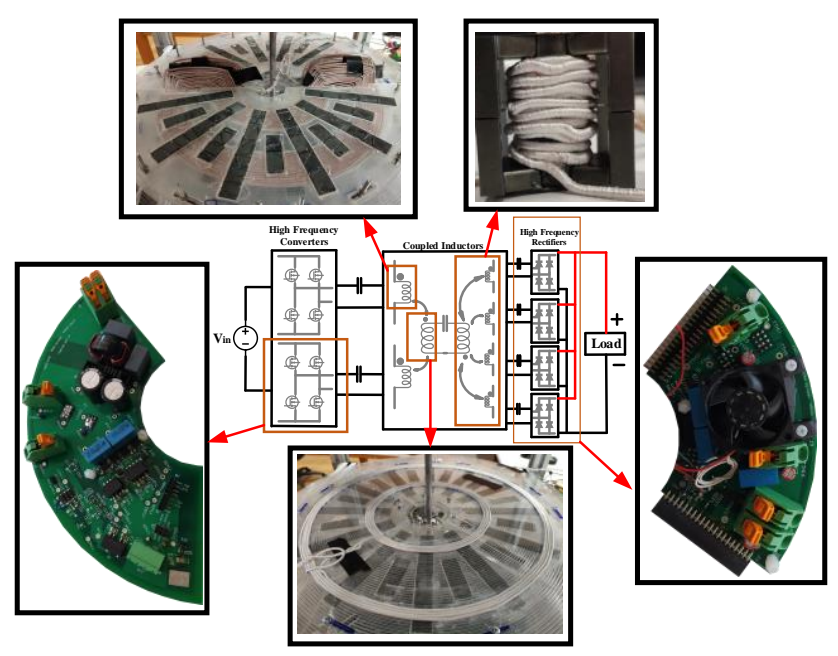

Fig. 5. Experimental setup for the proposed IPT system.

\section{B. IPT Coil Design Considerations}

In the previous section, a design methodology for the proposed topology was introduced. Although there are a few studies about the effect of coupling between the Tx and $\mathrm{Rx}$ coils [10], [11], the coupling between $\mathrm{Tx}$ and $\mathrm{Rx}$ coils
TABLE III

EXPERIMENTAL SETUP PARAMETERS

\begin{tabular}{ll} 
Tx side MOSFET & BSC600N25NS3G \\
Tx side Gate Driver & 2EDF7275F \\
Rx side Recrifier Diodes & C3D10060G \\
Litz Wire & $400 x 0.08 \mathrm{~mm}\left(2 \mathrm{~mm}^{2}\right)$ \\
Tx coil $D_{\text {in }}$ and $\mathrm{D}_{\text {out }}$ & $70-280(\mathrm{~mm})$ \\
Tx coil \# of turns & 13 \\
Tx coil inductances & $83.1-83.5(\mu \mathrm{H})$ \\
Resonator IPT coil Din and $\mathrm{D}_{\text {out }}$ & $65-280(\mathrm{~mm})$ \\
Resonator IPT coil \# of turns & 5 \\
Resonator IPT coil inductance & $23(\mu \mathrm{H})$ \\
$\mathrm{C}_{\mathrm{Tx}}$ & $13.6(\mathrm{nF})$ \\
$\mathrm{C}_{\mathrm{R}}$ & $15.21(\mathrm{nF})$ \\
$\mathrm{C}_{\mathrm{Rx}}$ & $44.2(\mathrm{nF})$ \\
Airgap & $10(\mathrm{~mm})$ \\
$\mathrm{f}_{0}$ & $150 \mathrm{kHz}$ \\
$\mathrm{f}_{\text {Ferrite shield }}$ & $155 \mathrm{kHz}$ \\
\hline
\end{tabular}

should be eliminated for the proposed design method. Since the air gap is under the designer's control, the resonator coil can be separated into two coils that are connected in series, where one coil couples the Tx and the other coil couples the Rx coils. With the addition of ferrite shielding between the resonator coil sections, the coupling between the $\mathrm{Tx}$ and Rx modules can be significantly reduced. However, it is important to note that the mutual inductances given in Table II will be harder to achieve with large air gaps. Therefore, the feasibility of such a system structure should be investigated with possible iterations based on the block diagram presented in Fig. 3.

In the proposed system structure, the resonator can be placed on both the rotating and stationary frame. Since the mutual inductance between the resonator and $\mathrm{Rx}$ coils is higher, the resonator coil is placed at the rotating frame. However, relatively lower mutual inductance and magnetic coupling between the $\mathrm{Tx}$ and the resonator coil results in a larger air gap.

The sizing of the ferrite core is highly dependent on the required level of fault tolerance. For open-circuited Rx module faults, the output power will be shared by the remaining Rx modules. Hence, choosing larger core provides safe operation under Rx module faults. After the initial design, PQ32x20 with N87 material with an air gap of $0.4 \mathrm{~mm}$ was found to be the minimum required magnetic core. Therefore, PQ35x35 with N87 material is selected as the magnetic core. The finalized HF coupled inductor (HFCI) parameters are presented in Table IV.

The resonator sides of the HFCI presented in Table IV are connected in series, and $53 \mu H$ was achieved for the $\mathrm{Rx}$ side resonator coil. Therefore for the Tx side, a coil with $L_{\text {self }}=21 \mu H$ and $L_{T x-R}=15.62 \mu H$ is designed.

\section{Experimental Results}

At this stage, it is essential to discuss the effect of rotation. The rated rotational speed is set to be $1500 \mathrm{rpm}(25 \mathrm{~Hz})$ with a resonant frequency of $150 \mathrm{kHz}$. Therefore for a complete rotation, there exists 6000 gate switching, which results in 
TABLE IV

PARAMETERS OF THE High FREQUENCY COUPLED INDUCTORS

\begin{tabular}{lllll} 
Parameters & $\mathrm{HFCI}_{1}$ & $\mathrm{HFCI}_{2}$ & $\mathrm{HFCI}_{3}$ & $\mathrm{HFCI}_{4}$ \\
\hline$L_{R}(\mu H)$ & 13.37 & 13.27 & 13.19 & 13.25 \\
$L_{R x}(\mu H)$ & 26.88 & 26.85 & 26.89 & 27.07 \\
$M(\mu H)$ & 18.05 & 18.14 & 18.01 & 18.09 \\
\hline \multicolumn{3}{c}{ Core Size } & \multicolumn{2}{c}{ PQ 35x35 } \\
Core Material & \multicolumn{2}{c}{$\mathrm{N} 87$} \\
Airgap & \multicolumn{2}{c}{$0.4 \mathrm{~mm}$} \\
Number of R Turns & \multicolumn{2}{c}{6} \\
Number of Rx Turns & \multicolumn{2}{c}{9}
\end{tabular}

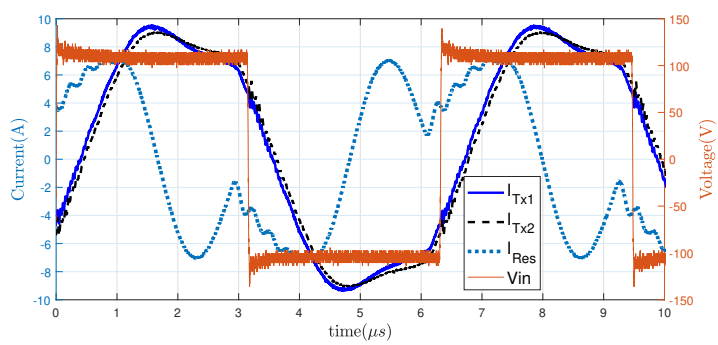

(a) $V_{i n}, I_{T x 1,2}$ and $I_{R}$ for normal operation.

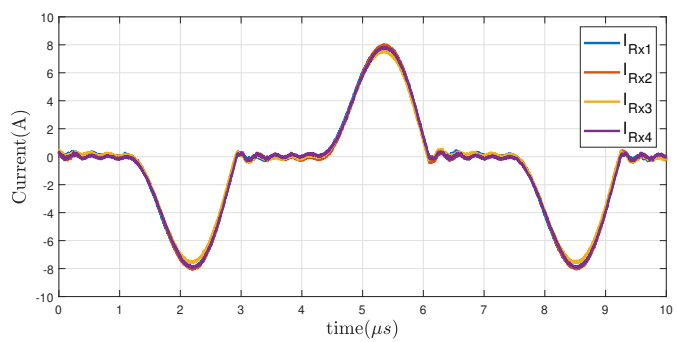

(b) Rx module currents for normal operation.

Fig. 6. Input voltage, Tx side currents, resonator current and Rx currents for no-fault operation. $\left(I_{T x 1,2} \approx 6.5 A_{r m s}, I_{R}=5.4 A_{r m s}\right)$

nearly 17 gate switchings for $1^{\circ}$ rotation. Moreover, the Tx and the resonator coil design satisfy constant $k_{T x-R}$, and hence the system can be assumed to be stationary regardless of the angular speed.

1) Normal Operation: Firstly, the system is operated at $156 \mathrm{kHz}$, slightly above the resonant frequency, which satisfies the zero voltage switching. The experimental results under normal operation are given in Fig. 6. The Rx currents are nearly equal and have a third harmonic component due to fullbridge diode rectifiers at the output stage. The reflected third harmonic component can also be seen at the resonator current.

In the analytical design, under $100 \%$ efficiency assumption, the Tx and resonator currents are taken equal. In the proposed system the resonator coil is divided into two parts where the coupling between the resonator and $\mathrm{Rx}$ side is achieved by HFCI's. Therefore, the length of litz wire used is much shorter than the Tx coils. Moreover, the HFCI's are slightly over-designed to satisfy the rated power transfer under single Rx module open circuit fault condition. Moreover, since ZVS operation is achieved, semiconductor losses are significantly reduced. Hence, it is safe to assume that the majority of the power loss occurs on the Tx side IPT coils. Therefore, unlike the analytical design, Tx currents are slightly higher than the resonator current.

2) Single and Double Rx Open Circuit Fault: In Fig. 7 a-d a single Rx open circuit fault is presented. As there are faulty $\mathrm{Rx}$ modules, the output power is shared between the remaining $\mathrm{Rx}$ modules. Therefore the power can either be reduced, which will result in a smaller system, or the Rx module can be over-designed and hence the output power can be kept at desired value under faulty conditions. Increasing the number of $\mathrm{Rx}$ modules will result in less stress on the remaining modules under faulty conditions; however, it also increases the chance of a semiconductor failure due to the increased number of semiconductors.

The proposed system is tolerant to receiver module faults. Under single Rx module open circuit faults, the Rx module currents are still nearly equal as shown in Fig. 7-d. The loss of a single Rx module resulted in a decrease of the reflected third harmonic component in the resonator current.

Then the effect of simultaneous faults in two Rx modules is investigated. The results are presented in Fig. 7b-e. The reflected third harmonic component reduced even further on both the Rx and resonator current. Since less modules are transferring power in this case, over-design rating of the $\mathrm{Rx}$ modules must be higher in order to supply rated output power.

3) Single Tx Open Circuit Fault: Finally, a single open circuit Tx fault is applied. Although the rating of each module is $500 \mathrm{~W}$, maximum of $250 \mathrm{~W}$ is transfered due to current limit as shown in Fig. 7c-f. Again the Rx module currents are nearly identical. The induced emf on the Tx side depends on the frequency, mutual inductance, and the secondary side current. Under an open-circuited Tx fault, the flux linkage is reduced by half, which also decreases the resonator current. Therefore, the induced emf on the Tx side is also halved, which results in high currents drawn from the source. Limiting the output power,the rated power under open-circuited Tx faults, the current rating of the Tx side power semiconductors should be increased. Another method is to increase the number of Tx modules, which will surely decrease the stress on the remaining modules during fault.

4) Open and Short Circuited Load Fault: The experimental results for open and short-circuited load conditions are also given in Fig. 8. In conventional series-series IPT systems, during open-circuited faults, the induced emf on the Tx side is theoretically zero, which results in dangerously high $\mathrm{Tx}$ side input currents. However, in the proposed system, it is the opposite. The induced emf on the resonator due to the $\mathrm{Rx}$ side becomes zero, and the intrinsic resistance of the resonator coil acts as the load. Assuming that the system operates near resonance frequency, the reflected impedance on the Tx side can be written as in (14).

$$
Z_{\text {ref }}=\frac{\omega^{2} M_{T x-R}^{2}}{j \omega L_{R}+\frac{1}{j \omega C_{R}}+R_{R}} \approx \frac{\omega^{2} M_{T x-R}^{2} \omega^{2}}{R_{R}}
$$

Therefore, the induced emf on the Tx side is high, which results in low input currents. The experimental results also 


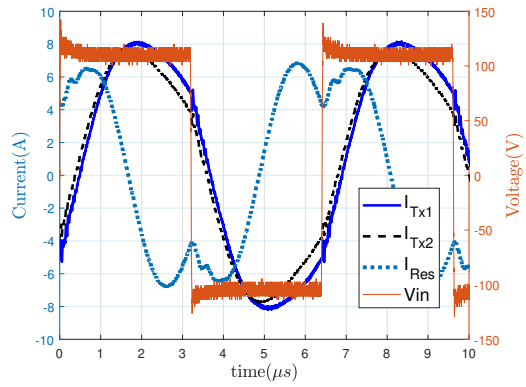

(a) $V_{i n}, I_{T x 1,2}$ and $I_{R}$ for single open-circuit $\mathrm{Rx}$ fault condition.

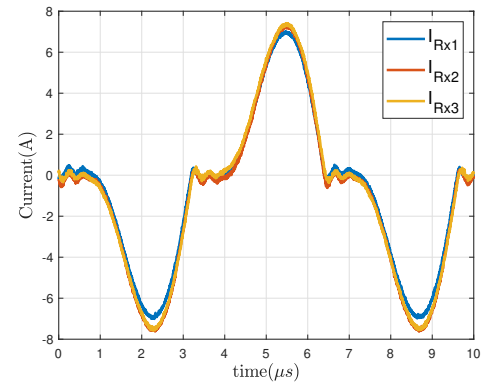

(d) Rx module currents for single open-circuit $\mathrm{Rx}$ fault condition.
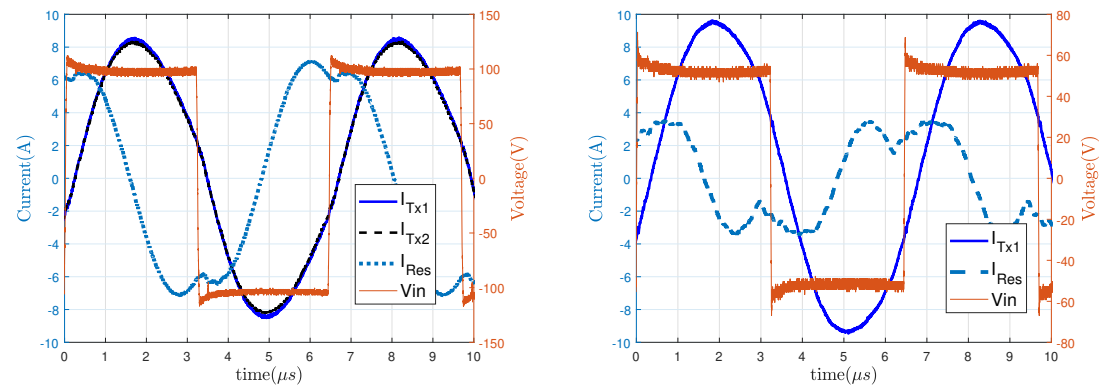
fault condition.
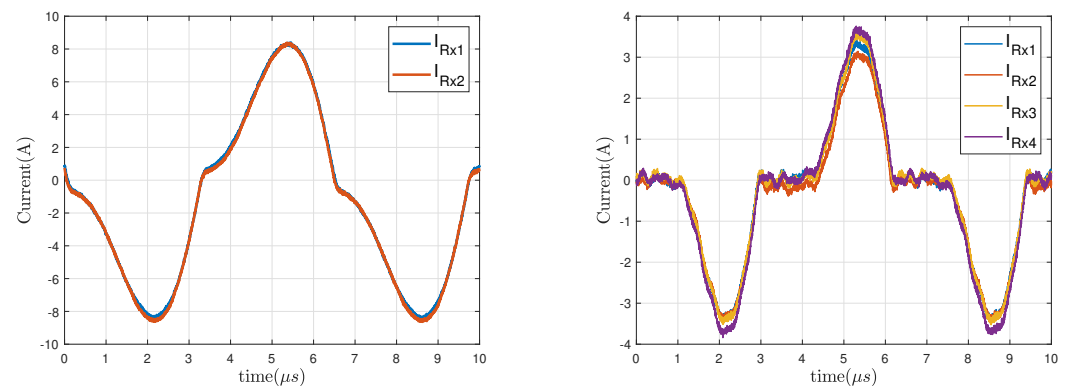

(e) Rx module currents for double open-circuit $\mathrm{Rx}$ fault condition.

(f) Rx module currents for single open-circuit $\mathrm{Tx}$ fault condition.

Fig. 7. Input voltage, Tx side currents, resonator current and Rx currents for single open circuited Rx, double open circuited Rx and single open circuited Tx faults.

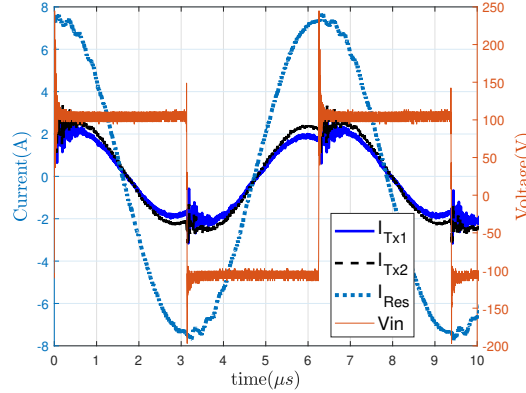

(a) Open circuit fault $V_{i n}, I_{T x}$ and $I_{R}$

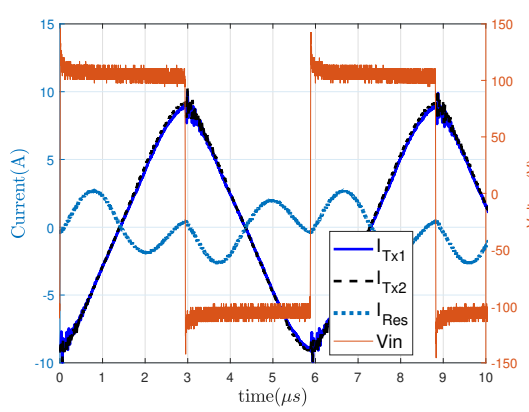

(b) Short circuit fault $V_{i n}, I_{T x}$ and $I_{R}$.

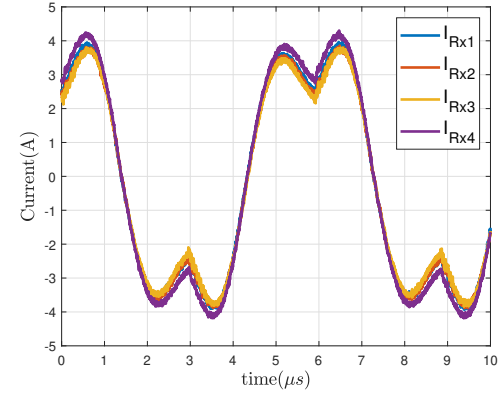

(c) Short circuit fault $I_{R x}$.

Fig. 8. Open and short circuit fault conditions. $V_{i n}, I_{T x}, I_{R}$ and $I_{R x}$ are shown for each type of fault. $f_{\text {operation }}=165 \mathrm{kHz}$

support the analytical discussion as given in Fig. 8-a. The short-circuited load condition is more challenging. The reflected impedance from the Rx to the resonator side becomes extremely high. Due to high impedance, resonator current reduces, and the reflected impedance on the Tx side due to the resonator becomes low. Therefore, dangerously high Tx input currents can be induced. The experimental results for open and short-circuited load condition are given in Fig. 8. At this stage, it is important to discuss a critical property of IPT systems with a middle resonator coil. Unlike conventional IPT systems, there exist three resonant frequencies, including the design resonant frequency. The upper frequency can be presented as in (15) where $L_{T x \text {,leakage }}$ is the leakage inductance of the Tx coil [12].

$$
\omega_{\text {upper }}=\frac{1}{\sqrt{C_{T x} L_{T x, l}}} \approx 175 \mathrm{kHz}
$$

The open and short circuit fault conditions are simulated in the frequency domain using the proposed system parameters and the results are presented in Fig. 9. During short-circuited load conditions, the quality factor of the input current gain increases significantly. Therefore, under the assumption of operation at slightly above the resonant frequency (to guarantee ZVS), the system is highly fault tolerant to short-circuited fault conditions.

Under open-circuited load condition, the situation is a bit different. Similar to the short circuit fault case, the quality factor of the input current increases significantly. However, for the higher resonant frequency, $\omega_{\text {upper }}$, the current gain increases significantly. Moreover, the input current starts to lead the input voltage, which results in the loss of ZVS operation. However, the magnitude of the input current drops which results in purely capacitive power drawn from the 

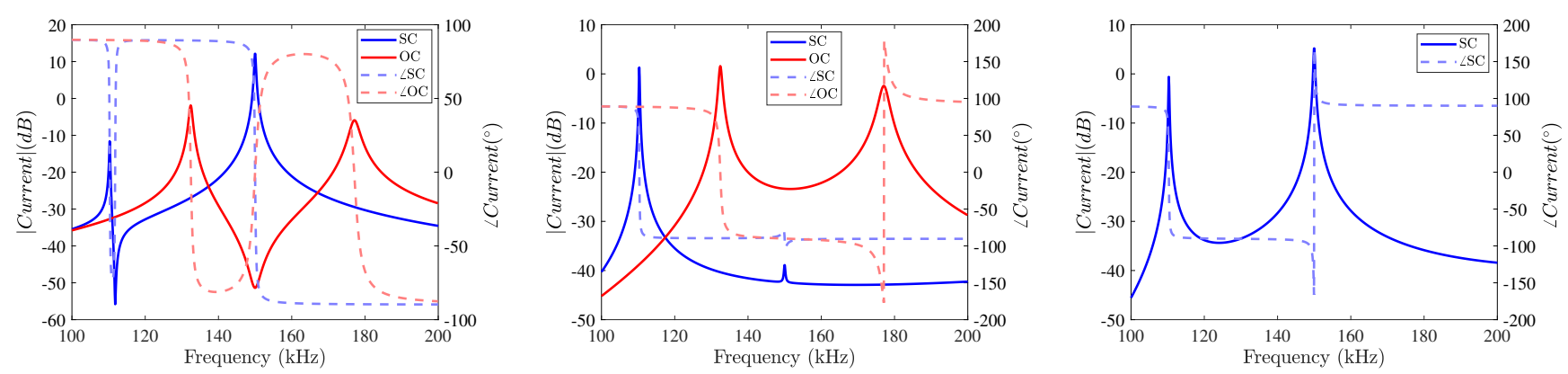

(a) Open and short circuit fault simulations for $I_{T x}$. (b) Open and short circuit fault simulations for $I_{R}$. (c) Open and short circuit fault simulations for $I_{R x}$.

Fig. 9. Open and short circuit fault condition simulation in frequency domain. $I_{T x}, I_{R}$ and $I_{R x}$ are shown for each type of fault.

TABLE V

EFFICIENCIES OF THE SySTEM UNDER DifFERENT FAULt CONDITIONS

\begin{tabular}{cccc} 
Operation & $\mathrm{P}_{\mathrm{in}}(\mathrm{W})$ & $\mathrm{P}_{\text {out }}(\mathrm{W})$ & Efficiency $(\%)$ \\
\hline Experimental & 1103.0 & 1000.0 & 90.9 \\
Single Rx open fault & 993.9 & 891.0 & 89.6 \\
Double Rx open fault & 988.8 & 885.5 & 89.5 \\
Single Tx fault & 296.9 & 242.4 & 81.6 \\
\hline
\end{tabular}

source with a magnitude less than the nominal real power. Although the system is tolerant to both open and short circuited load faults, it is not feasible to further transfer power under these fault conditions. These analyses show that even if there is a load fault, the results are not catastrophic, and the system can be turned off safely. The system is tested under various faults. The system efficiencies for the rated power of the system are given in Table V.

\section{DISCUSSION}

The experimental results show that the proposed design is highly tolerant to $\mathrm{Tx}$ and $\mathrm{Rx}$ side open circuit faults. However, few topics require special attention, which are the effect of modularity on the fault tolerance to open circuit Tx faults, modes of operation, and the origins of the third harmonic component in the Rx currents under normal and fault conditions.

\section{A. Effect of Modularity on Fault Tolerance}

Another critical topic is the discussion of the number of modules and maximum power transfer under a single $\mathrm{Tx}$ module fault condition. As stated in the experimental results, for a total of $1 \mathrm{~kW}$ system having two $500 \mathrm{~W}$ rated Tx modules, the maximum power which can be delivered under a fault condition is $250 \mathrm{~W}$ due to the current rating of the module. In Fig. 10, a theoretical approach for Tx module number, power rating per module, and maximum power transfer capability under single Tx module open-circuit fault is given. If it is desired to transfer rated power under a single Tx fault condition, it is feasible to increase the number of Tx modules. However, as the module number increases, the system sizing is problematic due to the reduced air gap to achieve the designed coupling between the Tx and the resonator coils. Moreover, the decrease of the intrinsic quality factor of the Tx modules will

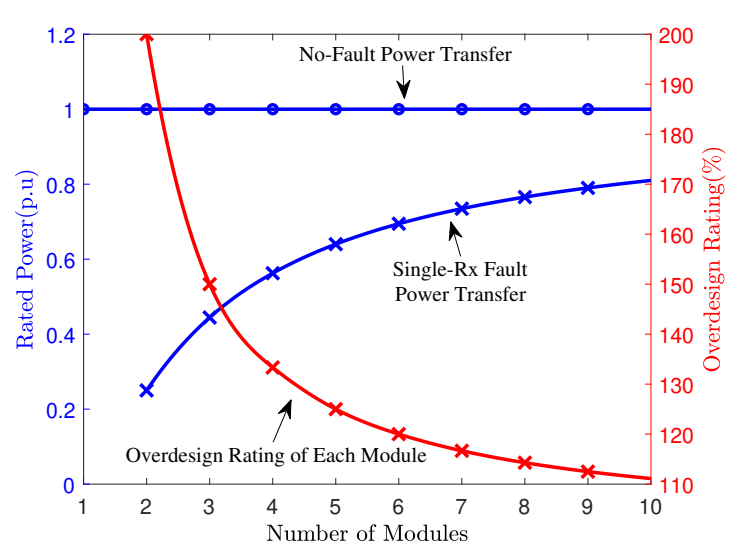

Fig. 10. Theoretical maximum power transfer for normal and single Tx side open circuit fault condition. Over-design rating of each Tx module to transfer the rated power under fault condition is also given.

reduce the overall system efficiency. Therefore, the number of Tx modules is highly dependent on the system requirements.

\section{B. Modes of Operation}

The proposed system has two separate modes of operation which are constant voltage (CV) and constant current (CC) point. The $\mathrm{CV}$ point is at the design resonant frequency $\left(f_{0}=150 \mathrm{kHz}\right)$ and the $\mathrm{CC}$ point is at the upper resonant frequency $\left(f_{\text {upper }}=187 \mathrm{kHz}\right)$. In Fig. 9, the zero phase angles are at $150 \mathrm{kHz}$ and $178 \mathrm{kHz}$ which overlaps with $\omega_{\text {upper }}$ given in (15). The slight differences between the simulation and experimental results are due to slight deviation of experimental parameter compared to the design parameters.

The operation frequency is swept over a wide range to achieve the voltage and current gain plots for different load conditions under CV and CC operations as shown in Fig 11. The data are taken for low voltage/power input since loss of ZVS results in increased switching loss. CV and CC operation is beneficial for battery charger applications. However, as also given in Fig. 9, under CV operation, the system is prone to short circuited load fault and the opposite is valid for CC operation. Therefore, the system should still have over-current protection. 


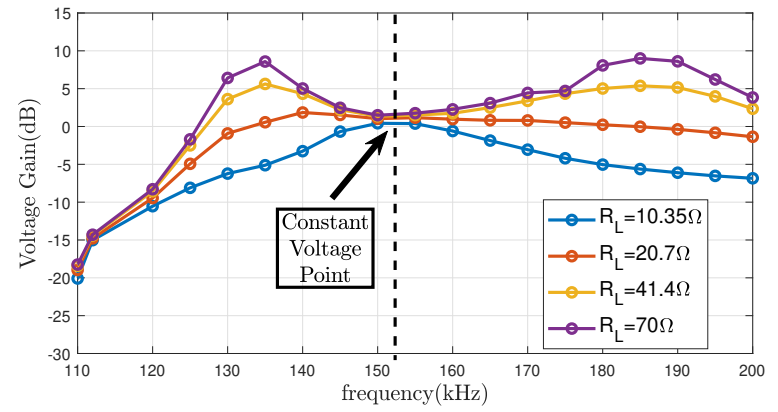

(a) Voltage gain for different loading conditions.

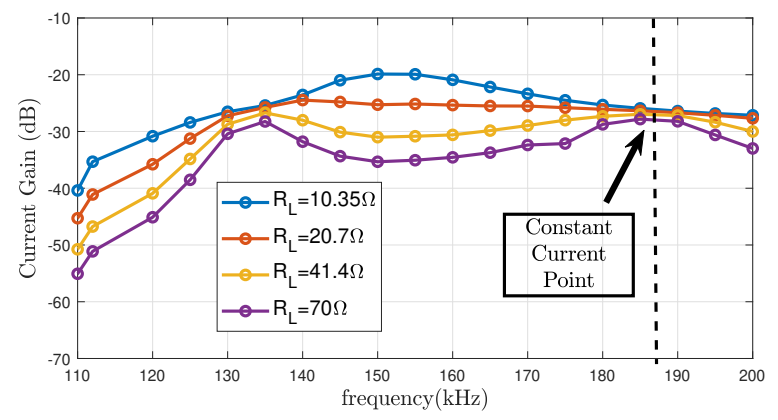

(b) Current gain for different loading conditions.

Fig. 11. Voltage and current gain plot for the experimental setup under different loading conditions. The $\mathrm{CV}$ and $\mathrm{CC}$ operation points are also presented.

\section{Third Harmonic Component}

In this study, the design methodology based on fundamental harmonic approximation (FHA) is presented under these assumptions: No-higher order harmonics and continuous coil current, also known as continuous current mode (CCM) [13]. However, an IPT system can also operate in discontinuous current mode (DCM) because of the diode rectifiers and load conditions [14]. In DCM, the current of the coils have odd harmonics $\left(3^{\text {rd }}, 5^{\text {th }}\right)$, which are comparable to fundamental component. Thus, the results may deviate from the FHA model. In this situation, the time-domain analysis (TDA) can be applied to consider the harmonics into account [15]. Although the system is designed usign FHA (considering CCM), we have experiment and simulation results having odd harmonics, which shows that the system is actually in DCM. However, a significant challenge is to obtain a bifurcation-free operation with the proposed modular design. The operation in DCM does not affect the bifurcation phenomenon and the power output. Thus, odd harmonics (especially the third) do not significantly distort the FHA assumptions, which can be used to obtain a bifurcation free system.

\section{CONClusion}

In this paper, a $1 \mathrm{~kW}$ novel series-series compensated common DC-bus modular IPT system with a middle stage resonator is introduced. A conventional series-series design methodology was modified in order to achieve a general design method for IPT systems with resonator coils. A design is proposed and tested for an IPT system that aims to replace slip rings and brushless exciters and to obtain reliable and speed independent operation. Modular structure is used to have fault tolerance to $\mathrm{Rx}$ and $\mathrm{Tx}$ side open circuit faults, which are the challenging fault types in conventional seriesseries IPT systems. Although, for fault tolerance to open and short-circuited load conditions changes for different operation frequencies, the system has a safe operation region between the design and upper frequency. During normal operation, the system has a $90.6 \%$ DC to DC system efficiency while delivering the rated power of $1 \mathrm{~kW}$. Unlike conventional seriesseries IPT systems, proposed design has fault tolerance to single-double Rx open circuit, single Tx open circuit, openshort circuited load conditions.

\section{REFERENCES}

[1] F. Zhang, S. A. Hackworth, Weinong Fu, and M. Sun, "The relay effect on wireless power transfer using witricity," in Digests of the 2010 14th Biennial IEEE Conference on Electromagnetic Field Computation, 2010, pp. 1-1.

[2] W. Zhong, C. K. Lee, and S. Y. R. Hui, "General analysis on the use of tesla's resonators in domino forms for wireless power transfer," IEEE Trans. Ind. Electron., vol. 60, no. 1, pp. 261-270, 2013.

[3] C. Sritongon, P. Wisestherrakul, N. Hansupho, S. Nutwong, A. Sangswang, S. Naetiladdanon, and E. Mujjalinvimut, "Novel ipt multi-transmitter coils with increase misalignment tolerance and system efficiency," in 2018 IEEE International Symposium on Circuits and Systems (ISCAS), 2018, pp. 1-5.

[4] J. Pries, V. P. N. Galigekere, O. C. Onar, and G. J. Su, "A 50-kW ThreePhase Wireless Power Transfer System Using Bipolar Windings and Series Resonant Networks for Rotating Magnetic Fields," IEEE Trans. Power Electron., vol. 35, no. 5, pp. 4500-4517, 2020.

[5] Y. Song, U. K. Madawala, D. J. Thrimawithana, and M. Vilathgamuwa, "Three-phase bi-directional wireless ev charging system with high tolerance to pad misalignment," IET Power Electronics, vol. 12, no. 10, pp. 2697-2705, 2019.

[6] D. Ahn and S. Hong, "Effect of coupling between multiple transmitters or multiple receivers on wireless power transfer," IEEE Trans. Ind. Electron., vol. 60, no. 7, pp. 2602-2613, 2013.

[7] G. Ke, Q. Chen, W. Gao, S. Wong, C. K. Tse, and Z. Zhang, "Research on ipt resonant converters with high misalignment tolerance using multicoil receiver set," IEEE Trans. Power Electron., vol. 35, no. 4, pp. 3697-3712, 2020

[8] H. Polat, E. Ayaz, O. Altun, and O. Keysan, "Balancing of common dc-bus parallel connected modular inductive power transfer systems," Sep 2020. [Online]. Available: https://www.techrxiv.org/ articles/preprint/Balancing_of_Common_DC-Bus_Parallel_Connected_ Modular_Inductive_Power_Transfer_Systems/13013882/2

[9] K. Aditya and S. S. Williamson, "Design guidelines to avoid bifurcation in a series-series compensated inductive power transfer system," IEEE Trans. Ind. Electron., vol. 66, no. 5, pp. 3973-3982, 2019.

[10] C. K. Lee, W. X. Zhong, and S. Y. R. Hui, "Effects of magnetic coupling of nonadjacent resonators on wireless power domino-resonator systems," IEEE Trans. Power Electron., vol. 27, no. 4, pp. 1905-1916, 2012.

[11] D. Ahn and S. Hong, "A study on magnetic field repeater in wireless power transfer," IEEE Trans. Ind. Electron., vol. 60, no. 1, pp. 360-371, 2013.

[12] S. Moon, B. Kim, S. Cho, C. Ahn, and G. Moon, "Analysis and design of a wireless power transfer system with an intermediate coil for high efficiency," IEEE Trans. Ind. Electron., vol. 61, no. 11, pp. 5861-5870, 2014.

[13] Y. Fang, B. M. H. Pong, and R. S. Y. Hui, "An enhanced multiple harmonics analysis method for wireless power transfer systems," IEEE Trans. Power Electron., vol. 35, no. 2, pp. 1205-1216, 2020.

[14] X. Qu, Y. Jing, J. Lian, S. Wong, and C. K. Tse, "Design for continuouscurrent-mode operation of inductive-power-transfer converters with loadindependent output," IET Power Electronics, vol. 12, no. 10, pp. 2458$2465,2019$.

[15] A. Safaee and K. Woronowicz, "Time-domain analysis of voltage-driven series-series compensated inductive power transfer topology," IEEE Trans. Power Electron., vol. 32, no. 7, pp. 4981-5003, 2017. 